\title{
AN ASSESSMENT OF RESPONSE, DIRECTION, AAD PLACE LEARNING BY RATS IN A WATER T-MAZE
}




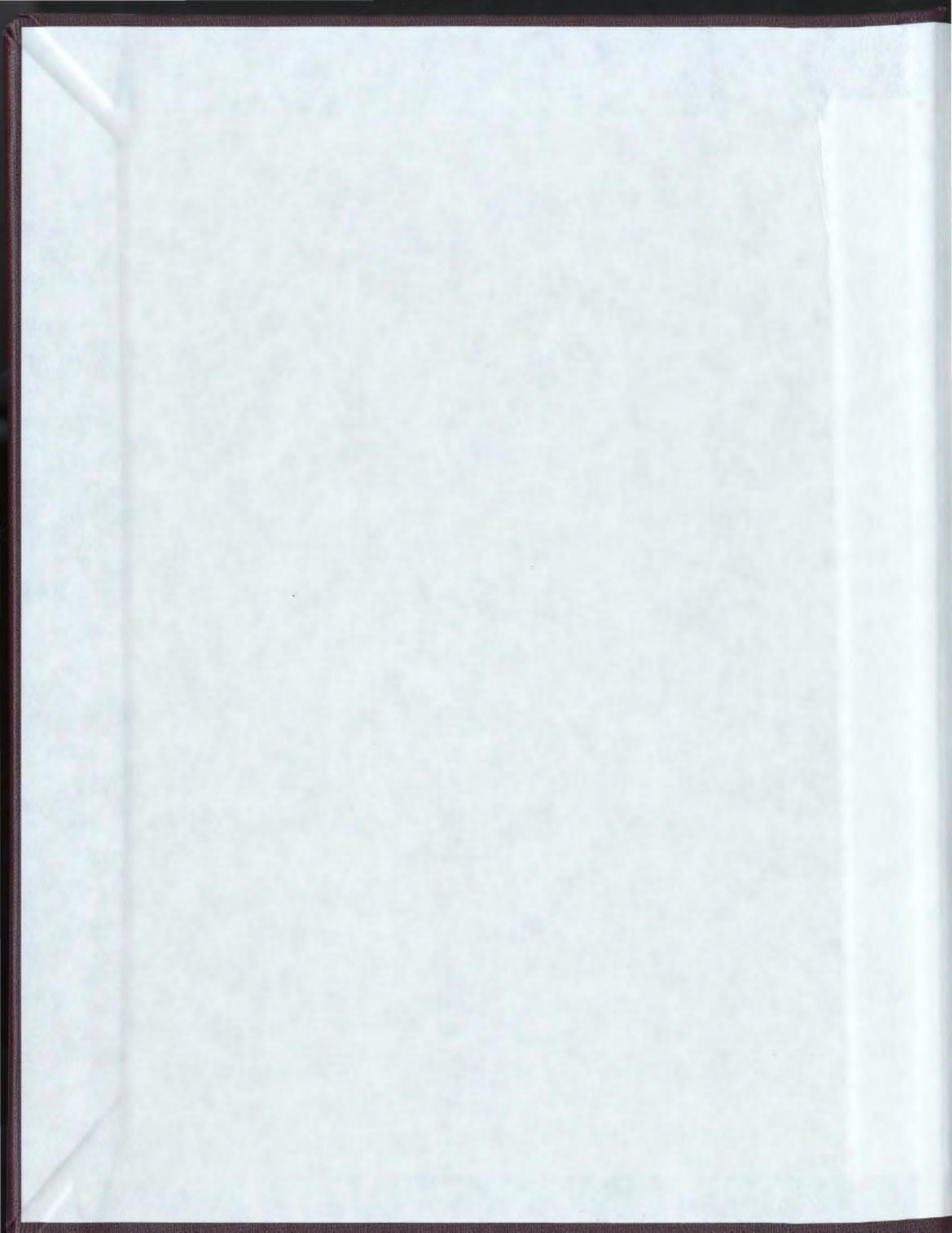





\title{
An Assessment of Response, Direction, and Place Learning by Rats in a Water T-maze
}

\author{
Jacqueline T. Whyte
}

\author{
A Thesis \\ Submitted to the Department of Psychology \\ In Partial Fulfillment of the Requirements \\ For the Degree \\ Master of Science
}

Memorial University of Newfoundland

St. John's, NL, Canada

2009 


\begin{abstract}
Behavioural data suggest that distinguishable orientations may be necessary for place learning even when distal cues define different start points in the room and a unique goal location. I examined whether changes in orientation are also important in place learning and navigation in a water T-maze. In Experiment 1, rats were trained to locate a hidden platform and given a no-platform probe trial after 16 and 64 trials with the maze moved to a new position. Direction and response strategies were more prevalent than a place strategy. In Experiment 2, acquisition of place, response, and direction strategies was assessed in a water T-maze that was moved between two locations during training. Rats were impaired on the place task when the maze was translated (moved to the $\mathrm{L}$ or R) but were successful when the maze was rotated across trials. These data are consistent with findings from appetitive tasks.
\end{abstract}




\section{Acknowledgements}

I would like to sincerely thank my research supervisor, Dr. Darlene M. Skinner, for her continued guidance and support throughout my research project, and for her comments and suggestions on multiple drafts of the current thesis. As well, thanks to my supervisory committee, Dr. Carolyn Walsh, and Dr. Gerard Martin for their input and feedback. I would also like to thank the multiple undergraduate students who helped in the lab, especially Scott Deibel, and Kate Murphy.

All research presented was funded by NSERC research grants awarded to Dr.

Darlene M. Skinner. 


\section{Table of Contents}

Abstract $\quad$ ii

Acknowledgements $\quad$ iii

Table of Contents iv

List of Figures

Introduction

The Cognitive Map 1

Place vs. Response Learning 2

Place Learning Redefined 4

Electrophysiology 5

Spatial Learning in an Aversive Task - The Morris Water Maze 6

The Present Study $\quad 8$

$\begin{array}{ll}\text { Experiment } 1 & 9\end{array}$

Methods 10

Results $\quad 12$

$\begin{array}{lr}\text { Experiment 2 } & 14\end{array}$

Methods 15

Results $\quad 17$

$\begin{array}{ll}\text { General Discussion } & 18\end{array}$

Future Directions $\quad 21$

$\begin{array}{ll}\text { References } & \mathbf{2 3}\end{array}$

$\begin{array}{lr}\text { Figure Captions } & 27\end{array}$

$\begin{array}{ll}\text { Figures } & 29\end{array}$ 


\section{List of Figures}

Figure 1: Schematic Representations of Tolman, Ritchie \& Kalish's (1946) Original Maze Positions

Figure 2: Schematic Representations of Maze Positions Differentiating Response, Direction, and Place Strategies

Figure 3: Mean ( \pm SEM) Trials Correct and Mean ( \pm SEM) Latency Over Blocks of 8 Trials for Experiment 1

Figure 4: Results from Experiment 1 Showing Arm Preference, First Choice, and Strategy Use Data

Figure 5: Results From Experiment 2, Mean ( \pm SEM) Trials to Criterion 


\section{An Assessment of Response, Direction, and Place Learning by Rats in a Water T-maze}

The question of what is learned when navigation to a particular location is reinforced is a point of debate. Two early hypotheses included learning the location relative to cues in the environment, or place learning, (O'Keefe \& Nadel, 1978; Tolman, Ritchie, \& Kalish, 1946), and learning the specific motor sequence(s) to arrive at the location, or response learning (Blodgett \& McCutchan, 1948; Hull, 1943). Much of the early research in the field of spatial navigation suggested that in environments with an abundance of cues, place learning was the dominant strategy (Restle, 1957; Tolman et al., 1946).

\section{The Cognitive Map}

In the 1940s, Tolman postulated the Cognitive Map theory in its most basic form. This theory stated that rats develop a map, or cognitive representation, of cues within their environment and the connections between these cues. The theory further proposed that navigation relied on the construction of maps and not on associations between stimuli and particular movements or actions, as had been previously proposed by Hull (1934a; 1934b). Hull's theory failed to adequately explain the complex patterns of animal behaviours or the flexibility of these behaviours (see Woodworth, 1938). Tolman's theory was more widely accepted because it more accurately depicted how an animal could navigate, and explained this flexibility.

Tolman's theory was based on the findings from a series of experiments carried out with rats on a plus maze with appetitive reinforcers. In one landmark study Tolman et al. (1946) trained rats on a T-maze to use a response strategy (i.e., always turn right) from two different start points, or a place strategy, in which the goal location did not 
change but the rat started from two different start arms, located $180^{\circ}$ from each other (See Figure 1). In both groups, rats were trained to a criterion of 10 successive errorless trials. In the Response group, only three of eight rats reached criterion in 72 trials, while all rats reached criterion in the Place group. Tolman et al. concluded that while both strategies could be learned with extended training, place learning was much more easily acquired than response learning.

O'Keefe and Nadel (1978) formalized Tolman's theory that animals form a cognitive representation of their environment. The Cognitive Map Hypothesis in its new form stated that a map was formed and stored in the hippocampus, and consisted of two major systems: a place system and a misplace system. The place system contains information about the organism's environment such as the location of objects and their spatial relations. The misplace system, however, signals changes which have occurred in the environment (i.e., the presence of a new object or the absence of an old object). Both systems work in conjunction to build new maps, or incorporate new information into old maps. Navigational strategies, or spatial learning, were also divided into two categories: taxon and locale systems of navigation. The taxon system of navigation included the guidance and orientation systems, and focused on local cue learning, including beacon learning (i.e., guidance) and response learning (i.e., orientation). The locale system, however, focused on the use of distal cues in the environment, and the rat's ability to use these cues as a navigational aid. The locale system was the primary focus of the Cognitive Map Hypothesis.

\section{Place versus Response Learning}

The notion that spatial strategies could be divided into two basic categories 
continued for decades. Using aspects of Tolman et al.'s (1946) original design, Packard and McGaugh (1996) assessed the relative contribution of place and response strategies to spatial learning by training rats on a T-maze to make a consistent turn to locate a food reward. Following 28 trials, animals were given a probe trial from a new start point located $180^{\circ}$ from the original start arm. If a rat trained to turn left also turned left on the probe trial, it was said to exhibit response leaming. If the rat turned right it was said to exhibit place learning because the right turn would take the rat to the original goal location. The rats were then given another 28 trials, followed by a second probe identical to the first. On the early probe, significantly more control rats chose the place arm over the response arm, while the opposite pattern was shown on the later probe. This pattern of results supported Tolman et al.'s work, indicating that a place strategy is the first strategy acquired, however, it also showed that with continued training rats switch strategies. In this experiment, rats with hippocampal or caudate nucleus inactivation were compared to sham operated controls. The results indicated that the hippocampus and the caudate nucleus mediated different neural mechanisms involved in place and response learning, respectively. This supported the Cognitive Map Hypothesis and the role of the hippocampus in spatial learning.

Packard and McGaugh's (1996) probe data suggested that early in training, animals utilize a place strategy while late in training a switch occurs and animal rely on a response strategy. Chang and Gold (2003), who trained rats to make a consistent response on a T-maze to obtain a food reward, also observed this shift from place learning to response learning with extended training. In their experiment, rats were given blocks of 20 training trials followed by a probe from a start point $180^{\circ}$ from the original 
start arm. Rats received 100 training trials with probes given after $20,40,6080$, and 100 trials. On the first probe, five of the six rats showed place learning over response learning, with the trend reversing for all other probes.

\section{Place Learning Redefined}

Despite the consistent findings of place learning superiority over response learning early in training, one major criticism of Tolman et al.'s (1946) work was not taken into account by later studies (i.e., Chang \& Gold, 2003; Packard \& McGaugh, 1996). Blodgett, McCutchen, and Mathews (1949) criticized Tolman et al. for not differentiating between a place strategy and a direction strategy. When animals in Tolman et al.'s experiment navigated to a fixed place, they did so from opposite sides of a plus maze. This resulted in animals going consistently in the same direction from the choice point to reach the goal (See Figure 1). Using aspects of Tolman et al.'s basic procedure, Blodgett et al. (1949) differentiated response, direction, and place strategies by moving the T-maze to four possible locations in the room. Each subject only received training from two positions. Rats were trained in one of seven groups (see Figure 2 (A-C, E - H): Place (Translated), Direction, Response, Place plus Direction, Place plus Response, Direction plus Response, and Direction plus Place plus Response. The Place group made significantly more errors than all other groups, while groups with a direction component common to both maze positions made the fewest number of errors. Based on the findings from this experiment, Blodgett et al. (1949) concluded that the superior place learning observed in Tolman et al.'s study might have been due to the rats using a direction strategy to locate the goal. 
Skinner et al. (2003) attempted to replicate the findings of Blodgett et al. (1949) using an open field and a T-maze. Rats were trained on a response, direction, or place problem to a criterion of eighteen correct trials out of twenty. The Place rats required significantly more trials to reach criterion than the Response group or the Direction group, and in fact, the majority of Place rats failed to reach criterion. These findings were consistent with those of Blodgett et al. (1949), and suggested that place learning, in the absence of a response or direction strategy, was very difficult.

Skinner et al. (2003) went on to test an altemative explanation for the poor place learning. They suggested that rats failed to solve the place problem when the maze was shifted left or right (translated) between trials because the rats failed to notice the change in the maze position or start point. The view from the two maze positions probably contained many overlapping features, preventing the rats from discriminating between start points. If the start points were more distinguishable, place learning should be easier. Skinner et al. tested this hypothesis by creating a second place problem where the maze was rotated between trials rather than translated (see Figure 2D). The results showed that rats learned this new place problem (rotation) as quickly as the response and direction problems. Further work has shown that discriminable start points (Horne, Martin Harley, \& Skinner, 2007) and changes in initial orientation at the start points (Skinner, Horne, Murphy, \& Martin, in press) lead to success in a variety of spatial tasks.

\section{Electrophysiology}

An early cell-recording study using different local mazes in the center of the same room with the same distal cues suggested that it was the local maze, not the distal room cues, that controlled place cell firing (Kubie \& Ranck, 1983). Converging evidence from 
behavioural and electrophysiological studies indicate the importance of apparatus boundaries, in addition to distal cues, in spatial learning (Hamilton, Akers, Weisend, \& Sutherland, 2007; Hamilton et al., 2008; Horne, Martin, Harley, \& Skinner, 2007;

Knierim \& Rao, 2003; Siegel, Rao, Lee, \& Knierim, 2005; Skinner et al., 2003; Stringer, Martin, \& Skinner, 2005; Walsh, Harley, Corbett, Skinner, \& Martin, 2008;

Yoganarasimha \& Knierim, 2005). Recent studies have shown that when an apparatus location was translated by $33-120 \mathrm{~cm}$, relative to distal cues, the firing of the majority of place cells was determined by the rats' location on an apparatus rather than by the rats' location in the room (Knierim \& Rao, 2003; Siegel et al., 2005; Yoganarasimha \& Knierim, 2005). When the distal visual cues were rotated, place fields were correspondingly rotated, as were head direction cells (Yoganarasimha \& Knierim, 2005). Thus, when the maze is translated, place cells remain tied to the animal's location on the apparatus, and do not signal a change. Rotation, however, causes a shift in the place fields, indicating that the change in surroundings is noted.

\section{Spatial Learning in an Aversive Task - The Morris Water Maze}

Morris (1981) investigated place learning and provided evidence of cognitive maps in rats in his landmark experiments using a water maze. Rats were trained in an open field water maze to locate a hidden platform using distal cues in the room; the platform remained in the same location in the room relative to these cues, but the start point for the rat was variable. With increasing numbers of trials, rats learned to locate the platform from any start point by navigating in a relatively straight line from the start point to the platform. Control rats were impaired at locating the submerged platform if it did not remain in a fixed location. This suggests that the animals were not using a 
guidance system of navigation, but a cognitive map that enabled them to move directly to the submerged platform based on distal cues in the room. On subsequent probe trials, when the fixed platform was removed from the pool, rats spent significantly more time in the quadrant of the pool where the platform had previously been located.

Hamilton, Akers, Weisend, and Sutherland (2007) conducted an experiment using a Morris water maze to determine whether rats use the distal cues in the room to navigate to a specific location or to swim in a certain direction. Rats were trained to locate a hidden platform in a pool from multiple start points. Following training, the platform was removed and the rats were given a probe trial with the pool in the same position or with the pool moved to a new position, such that the absolute location of the platform was in the opposite quadrant relative to the boundaries of the pool. On probe trials rats did not swim to the absolute location of the platform, but rather swam in the direction that had been reinforced in training. Thus, the rats went to the relative location of the platform and searched at the appropriate distance from the pool wall. The authors concluded that rats use distal cues to provide directional information and the apparatus boundaries to provide distance information.

Hamilton et al. (2008) went on to examine spatial learning when the pool wall was removed as a prominent cue. As in previous experiments, rats were trained to locate a hidden platform from varying start points during training. On a subsequent no platform probe trial, the maze either remained in the same position, or was shifted such that the relative location of the platform in the pool differed from the absolute location of the platform in the room. With transparent pool walls, the animals in both groups showed a preference for the absolute location of the platform over the relative location of the 
platform. This suggests that with the elimination of the apparatus boundaries as a prominent cue, animals could use the distal cues to travel to a specific point in space. In a recent follow-up to that study, Hamilton et al. (2009) have shown that the preference for the absolute location over the relative location is a transient effect. Thus, most of the evidence from studies where the apparatus is shifted suggests that animals use distal cues to travel in a particular direction and not to a specific point in space.

To fully assess the prevalence of the navigational strategies previously studied using dry-land mazes with appetitive reinforcers in the aversive water maze task, it will be necessary to examine the acquisition and use of response, direction and place strategies by rats in a water plus maze.

\section{The Present Study}

Behavioural experiments have shown that place learning is impaired, relative to response and direction learning, when a single maze (open field, T-maze, or plus maze) is moved between two adjacent locations (Blodgett et al., 1949; Skinner et al., 2003; Stringer et al., 2005; Walsh et al., 2008). For example, in the Skinner et al. (2003) study, rats were required to make a right turn when the maze was in the left location but a left turn when the maze was translated to the right position, to locate the food reward, which was fixed relative to distal cues. Rats had difficulty solving this place problem and were significantly impaired relative to a Response group that simply made a left turn regardless of maze position, or a Direction group that moved in a constant direction to locate food (See Figure $2(\mathrm{~A}-\mathrm{D})$ for a schematic of the maze positions).

To determine whether initial orientation is important in place learning and navigation across spatial tasks and motivational conditions, a series of experiments using 
a water task were conducted. Consistent with earlier findings using an appetitive task (Skinner et al., 2003), a preference for directional responding by rats in a water task has been reported across a variety of conditions but always in a more open maze configuration with no obvious response solution (Hamilton et al., 2008; Hamilton et al., 2007). Thus, in the current thesis I assessed response, direction, and place strategies in a water T-maze. While previous work has shown that animals can learn response, direction, and place solutions equally on an appetitive task; if the maze is rotated rather than translated in the place task (Skinner et al., 2003), it is unknown which solution the animals adopt when alternate strategies are available and with different levels of training. Previous studies using appetitive reward in plus mazes have shown that rats often switch strategies with extended training (Chang \& Gold, 2003; Packard \& McGaugh, 1996).

\section{Experiment 1}

In the first experiment all rats were trained to make a left or right turn to locate the hidden platform, and then given a no platform probe trial immediately after trials 16 (early) and 64 (late) to determine the strategy used to locate the platform and whether this strategy changed with extended training (Chang \& Gold, 2003; Packard \& McGaugh, 1996). Animals were divided into four groups designed to differentiate between response, direction and place strategies (see Figure 2 (A-D)). In the Translation group, the maze and the rats' start point were shifted left (or right) of the training position; this test was an unambiguous test of place learning but confounded response and direction strategies. In the $90^{\circ}$ Rotation group, the strength of response and place strategies was assessed in the absence of a direction strategy when the maze was rotated on probe trials. In the $180^{\circ}$ Rotation group, the start point was on the opposite side of the maze; this was 
an unambiguous test of response learning but confounded place and direction strategies. In the Translation plus $180^{\circ}$ Rotation group, the maze was translated and the start point was rotated $180^{\circ}$; this was an unambiguous test of direction learning but confounded place and response strategies.

\section{Method}

Subjects: Thirty-three naìve, male, Long Evans rats, obtained from Charles River Company (St. Constant, Quebec, Canada), and weighing $216 \mathrm{~g}-267 \mathrm{~g}$ at the start of the experiment were used. Rats were housed in clear plastic cages $(45 \times 25 \times 21 \mathrm{~cm})$ with secured metal lids and maintained on a 12-hr/12-hr light/dark cycle with lights on at 0800. All rats had continuous access to food and water in their home cages. Animal care and all procedures used in the present experiments were approved by Memorial University's Institutional Committee on Animal Care and followed the Canadian Council on Animal Care guidelines.

Apparatus. The training room $(850 \times 680 \mathrm{~cm})$ had windows covering much of the south and west walls. The east wall contained a chalkboard $(275 \times 180 \mathrm{~cm})$; the north wall contained a door and was lined with cabinets and counters. The northeast corner of the room contained a sink and the south and west walls contained stacks of wooden tables and chairs.

The water maze consisted of a plus maze set inside a metal circle $(178 \mathrm{~cm}$ diameter x $52 \mathrm{~cm} \mathrm{high).} \mathrm{Clear} \mathrm{Plexiglas} \mathrm{walls} \mathrm{(} 52 \mathrm{~cm}$ high) formed four radiating arms that opened into a center circular area. The walls of the arms were $62 \mathrm{~cm}$ in length with a width of $33 \mathrm{~cm}$ at the end and $11 \mathrm{~cm}$ at the center circle, which was $52 \mathrm{~cm}$ in diameter. Located at the end of each arm was a metal platform $(21 \times 9 \times 21 \mathrm{~cm})$ that could be raised 
and lowered by pulling a stainless steel wire attached to the platform. The end of the wire was pushed through a small opening in the wall of the maze so that the wire could be manipulated from outside the maze. The plus maze was converted to a T-maze by inserting a metal barrier in front of the arm directly opposite the start arm. The water in the maze was $24 \mathrm{~cm}$ deep with the surface $2-3 \mathrm{~cm}$ above the escape platform. The water was made opaque by the addition of approximately $250 \mathrm{ml}$ of non-toxic white Tempura paint (Rich Art Color Company, Northvale, NJ). Water temperature was equilibrated with room temperature (approximately $20^{\circ} \mathrm{C}$ ).

Training: Seventeen rats were trained to make a left turn and sixteen to make a right turn from a constant start arm to locate a hidden platform. The rats were brought into the training room in groups of four and placed individually in plastic holding cages that were similar to the home cages. On each trial, a rat in its holding cage was brought to a chair positioned at the start arm. The rat was placed in the start arm facing the wall of the maze. The arms visited by the rat and the time (in seconds) taken to locate the hidden platform were recorded. A rat was considered to have made a choice when the body, minus the tail, was inside the arm. A correct trial was one where the rat entered the arm containing the platform, and successfully climbed onto the platform, without entry into other arms. Once the rat located the platform, it was allowed to sit there for $10 \mathrm{~s}$ before being removed from the maze. If the rat did not locate the platform in $60 \mathrm{~s}$, it was placed on the platform by the experimenter. The experimenter remained at the start arm for the duration of the trial. Upon completion of the trial, the rat was placed back in the cage and carried back to the holding table and the next rat began its trial. The rats received four training trials on day 1 , eight training trials on day 2 , and four training trials on day 3 , 
followed by a no-platform probe trial from a novel location. The inconsistency in the number of trials per day was due to the fact that training took a long time on the first day since the rats were not given a habituation swim in the water task. This also meant that the rats had no way of predicting the probe trial as it was the $5^{\text {th }}$ trial and they had received 8 trials the previous day. The rats were then given a one-day break followed by an additional 48 trials ( 8 trials/day), for a total of 64 training trials. Immediately after trial 64 , the rats were given a second probe trial, identical to the first probe. Thus, the rats received 66 trials in total; 64 training trials and 2 probe trials.

Probe Trials: Upon completion of the first 16 trials the rats were divided into four groups for the probe trials, with an equal number of left and right turning rats in each group (with the exception of the $90^{\circ}$ Rotation group that had nine subjects). The training and probe positions are outlined in Figure 4. For each probe trial, the first choice and time spent in each arm were recorded. Following $60 \mathrm{~s}$, the animal was removed from the water and returned to its cage. The Translation group $(n=8)$ was probed from a position to the left or right of the original start point; the $180^{\circ}$ Rotation group $(n=8)$ was probed from a position directly opposite the original start arm; the Translation $+180^{\circ}$ Rotation group (n $=8$ ) was probed from a position opposite the original start arm and shifted right or left; and the $90^{\circ}$ Rotation group $(\mathrm{n}=9)$ was probed from a position to the left or right and rotated such that the new start point was at a right angle to the original start location. The same probe trial was repeated following trial 64 .

\section{Results}

Rats were divided into groups based on performance during the first 16 trials. A two-way (Group x Trials) ANOVA comparing trials correct over blocks of eight trials 
revealed only a main effect of Trials $(F(7,203)=8.96, \mathrm{p}<.05)$ (Figure 3, top panel). The effect of trials is due to improvement over blocks of trials as evidenced by a significant linear trend $(F(1,29)=13.15, p<.05)$. Similarly, a two-way ANOVA on latency to locate the hidden platform revealed only a main effect of Trials $(F(7,203)=$ 21.57, $\mathrm{p}<.05$ ) (Figure 3, bottom panel). Again, this effect of trials is due to improvement over blocks as evidenced by a significant linear trend $(F(1,29)=44.03, p<$ $.05)$.

One probe trial was given early in training, before asymptote was reached, while the second was given late in training, after the rats had reached asymptote. T-tests were used to compare performance on the second block of training trials with performance on the last block of training trials. The comparisons revealed a significant difference between the two blocks for both trials correct $(\mathrm{t}(32)=3.94, \mathrm{p}<.05)$ and latency $(\mathrm{t}(32)=$ $4.11, \mathrm{p}<.05$ ), indicating an improvement. The probe trials revealed little evidence of place learning. Neither of the two groups that were given an unambiguous test of place learning spent more time in the arm associated with the correct place. In the Translation group, the rats spent more time in the arm associated with the correct response and/or direction than in the place arm after $16(t(1,14)=4.23, \mathrm{p}<.05)$ and 64 trials $(t(1,14)=$ $3.46, \mathrm{p}<.05$ ) (see Figure 4). A rat's first choice and the arm where it spent the most time were not always the same. In the first probe trial equal numbers of rats chose the place arm and the response + direction arm as their first choice, but all rats spent more time in the response + direction arm. By the second probe, all rats chose the response + direction arm first. In the $90^{\circ}$ Rotation group the rats spent more time in the arm associated with the correct response on both the first $(t(1,16)=5.47, \mathrm{p}<.05)$ and the second $(\mathrm{t}(1,16)=$ 
4.33, $\mathrm{p}<.05$ ) probe trials (see Figure 4). In this group, the first choice data were consistent with the time spent in the arms as $8 / 9$ and $9 / 9$ rats chose the response arm on the first and second probe trials, respectively.

In the remaining two groups, the $180^{\circ}$ Rotation and Translation $+180^{\circ}$ Rotation groups, a place strategy was confounded with a direction or response strategy. Given the lack of evidence for a place strategy in the Translation and $90^{\circ}$ Rotation groups, it seems reasonable to assume that any preferences observed in the $180^{\circ}$ Rotation and Translation $+180^{\circ}$ Rotation groups were attributable to the other strategy (i.e., direction or response). Despite the fact that $6 / 8$ rats in both groups chose the arm associated with the correct response on the first probe trial, neither the $180^{\circ}$ Rotation group $(t(1,14)=.487, \mathrm{p}>.05)$ nor the Translation $+180^{\circ}$ Rotation group $(\mathrm{t}(1,14)=1.29, \mathrm{p}>.05)$ spent more time in the response or direction arm on this test (see Figure 4). By the second probe trial both the $180^{\circ}$ Rotation group $(\mathrm{t}(1,14)=7.36, \mathrm{p}<.001)$ and the Translation $+180^{\circ}$ Rotation group $(t(1,14)=2.81, \mathrm{p}<.05)$ spent significantly more time in the arm associated with the correct direction. First choice data were again not always consistent with the time spent in the arms as equal number of rats in the Translation $+180^{\circ}$ Rotation group chose the response and direction arm as their first choice but spent more time in the direction arm. In the $180^{\circ}$ Rotation group, $7 / 8$ rats chose the direction arm first.

\section{Experiment 2}

Consistent with the findings from previous T-maze experiments (i.e., Blodgett et al., 1949; Skinner et al., 2003) the arm preference data from Experiment 1 revealed that response and direction solutions are more prevalent than a place solution. Even when the apparatus was rotated there was little evidence of a place strategy, despite research 
indicating that rats solve the rotation place problem as easily as the response or direction problem (Skinner et al., 2003). In a further attempt to examine place learning in a water T-maze, rats were trained on an acquisition task where the maze was moved between two locations during training as in earlier experiments using appetitive tasks (Skinner et al., 2003; Skinner et al., in press; Stringer et al., 2005; Walsh et al., 2008). In a pilot experiment, it was shown that acquisition of the translation place problem was difficult in the water maze. However, Hamilton et al. (2008) demonstrated a preference for place navigation over directional responding using the open maze configuration when the pool wall was removed as a prominent cue. Consequently, in Experiment 2 acquisition of place learning was assessed in a new water T-maze with transparent Plexiglas walls. In addition to the translation and rotation place problems used previously (Skinner et al., 2003), in this experiment the performance of rats trained on a place task when the place strategy was combined with a response or direction strategy was also assessed.

\section{Method}

Subjects: Sixty-five naïve, male, Long Evans rats, obtained from Charles River Company (St. Constant, Quebec, Canada), and weighing between $175 \mathrm{~g}-217 \mathrm{~g}$ at the start of the experiment were used. Animals were housed and maintained as in Experiment 1. Apparatus: The training room $(528 \times 464 \times 267 \mathrm{~cm})$ had windows covering the north wall, and two doors, one located on the south wall, one on the east. In the southwest corner of the room was a sink; shelves lined the west wall, and half of the east wall. The southeast corner of the room contained stacked boxes and a coat rack, as well as two garbage cans. Animal cages were arranged on a table below shelving on the west wall. 
tank ( $120 \mathrm{~cm}$ in diameter, $31 \mathrm{~cm}$ high), which was mounted on a metal frame with wheels. Plexiglas walls extended $31 \mathrm{~cm}$ above the metal tank, as did the Plexiglas plus insert. The arms of the plus measured $11.5 \mathrm{~cm}$ wide, and $52.5 \mathrm{~cm}$ long. Water level was maintained at approximately $2.5 \mathrm{~cm}$ below the top of the metal tank, and water temperature was equilibrated with room temperature (approximately $20^{\circ} \mathrm{C}$ ). Water was made opaque by the addition of approximately $250 \mathrm{ml}$ of non-toxic white Tempura paint (Rich Art Color Company, Northvale, NJ). Platforms consisted of a length of plumbing tubing (white) filled with sand, capped, and topped with a non-slip drawer liner; a rectangular Plexiglas base prevented the platform from tipping. The platform could be manually lifted and shifted to the ends of various arms as needed, and rested $1-2 \mathrm{~cm}$ below water level. The plus was converted into a T-maze using a section of clear Plexiglas which could be snapped to the plus using butterfly clips, obstructing physical access to the arm opposite the start arm, but not obstructing visual access.

Training: Animals were divided into eight groups based on body weight and were trained to locate a hidden platform from two maze positions (see Figure 2 for maze positions). The Response group ( $n=8$ ) was trained to make a consistent turn (half went left, half went right), the Direction group $(n=8)$ was trained to go in a consistent direction (half went west, half went right), in the Translation group $(n=8)$ the maze was translated to the left or right during training while in the Rotation group $(n=8)$ the maze was rotated by $90^{\circ}$ across trials. The remaining four groups were combination groups, which confounded strategies. The Place + Direction group $(n=8)$ was trained to go to the same place in the room and always approached this place from the same direction (half went west, half went east). The Place + Response group $(n=8)$ was trained to make a 
consistent response (half went right, half went left) and the response took the animal to the same place in the room. The Response + Direction group $(n=8)$ confounded direction and place such that the animal always went in the same direction and made the same response to reach the platform (i.e. left and west, right and east, etc.). The final group, Response + Direction + Place $(n=9)$, confounded all three strategies with the animals consistently going in the same direction by making the same response to go to the same place. This group started from a single start point, unlike the others, in order to ensure all strategies were confounded. For each group with a place component, the hidden platform was in the same location, relative to extra-maze cues, on each trial. Training procedures were the same as those used in Experiment 1, except no more than two trials in a row were given from the same start position. Animals were given four trials per day for the first two days, then eight trials per day for subsequent training days until a criterion of 18 correct trials out of 20 was reached. If animals had not reached criterion by trial 136, training was stopped.

\section{Results}

All rats in the Response, Rotation, Response + Place, Direction + Place, and Response + Direction + Place groups reached criterion in under 136 trials. Six of the eight rats in each of the Direction and Response + Direction groups reached criterion while only $1 / 8$ rats in the Translation group reached criterion in that same period. A oneway ANOVA revealed a significant effect of group $(F(7,57)=23.81, p<.01)$ (see Figure 5). Post-hoc Bonferroni tests revealed that the Translation group required more trials to reach criterion than the other six groups (p's $<.05$ ), which did not differ (p's > $.05)$. This pattern of data replicates previous findings using appetitive tasks (Skinner et 
al., 2003; Skinner et al., in press; Stringer et al., 2005; Walsh et al., 2008), suggesting that spatial strategies are similar across different motivational conditions.

\section{General Discussion}

Rats do not adopt a place strategy to locate a hidden goal when alternate strategies (i.e., response and direction strategies) are available. Neither of the groups given unambiguous tests of place learning in Experiment 1 showed a preference for the arm associated with the correct place. The Translation and $90^{\circ}$ Rotation groups spent more time in the arm associated with the correct response and/or direction than in the arm associated with the correct place. These data are consistent with previous experiments showing that response and direction strategies were learned more readily than place strategies on appetitive tasks (Blodgett et al., 1949; Skinner et al., 2003; Skinner et al., in press; Stringer et al., 2005; Walsh et al., 2008) and with experiments showing a preference for navigation based on direction over place navigation in the water maze (Hamilton et al., 2007; 2008).

Early in training response solutions appear to compete with direction solutions, as evidenced by the lack of arm preference in the $180^{\circ}$ Rotation and the Translation $+180^{\circ}$ Rotation groups on the first probe trial of Experiment 1. Later in training, direction solutions are more prevalent if they are available. Both the $180^{\circ}$ Rotation and the Translation $+180^{\circ}$ Rotation groups spent more time in the arm associated with the correct direction than in the arm associated with the correct response on the second probe trial.

Previous findings using an appetitive task have shown that rats exhibit a place strategy early in training and a response strategy with continued training (Chang \& Gold, 
2003; Packard \& McGaugh, 1996). Rats in the $180^{\circ}$ Rotation group were given probe trials from the same position as rats in the Packard and McGaugh (1996) and Chang \& Gold (2003) studies. In the present study, more rats chose the response arm as the first choice early in training but went on to distribute their time equally between the two arms over the $60 \mathrm{~s}$ test. Later in training, when rats in the earlier studies exhibited response learning, there was a significant preference for the Place + Direction arm in the present experiment, both in terms of rats' first choice and time spent in the arms. Whether the different pattern of results across the studies reflects differences in procedural details remains to be determined. For example, time spent in the two arms was not recorded in the earlier, appetitive tasks.

Consistent with previous studies showing a preference for directional responding in aversive tasks (Hamilton et al., 2007; 2008), rats in the $180^{\circ}$ Rotation and the Translation $+180^{\circ}$ Rotation groups spent more time in the arm associated with the correct direction on the probe trial given late in training. On the earlier probe trial, response and direction strategies were equivalent. Although rats exhibited a directional strategy both early and late in training in the Hamilton et al. $(2007 ; 2008)$ studies, those experiments were conducted in an open water maze with no obvious response solution.

As seen in earlier experiments using appetitive tasks (Skinner et al., 2003; Skinner et al., in press; Stringer et al., 2005; Walsh et al., 2008), the results from Experiment 2 confirm that place learning is difficult in the absence of a response or direction strategy (Translation group) unless there is a change in orientation across the two maze positions (Rotation group). The data from Experiment 2 are also consistent with Blodgett et al.'s (1949) original findings that a place component, when combined with other strategies, 
does not improve performance. Rats in the Place + Direction and Place + Response groups did not solve the spatial task in fewer trials than the Direction or Response groups. Surprisingly, rats in the Response + Direction group did not perform better than rats in the Response or Direction groups. Perhaps the individual spatial solutions are not additive.

In contrast to earlier findings by Hamilton et al. (2008), making the pool walls transparent did not encourage place learning when the maze was translated. The absence of a place strategy in the water T-maze may be due to the dominance of response and direction solutions in an apparatus with confined movement trajectories. However, more recent work from Hamilton's group has shown that the place learning observed with the transparent pool wall is a transient effect (Hamilton et al., 2009). The rats in this later study showed place learning after 12 training trials with a preference for directional responding emerging in as little as 24 trials. It remains to be seen whether a preference for a place strategy will be observed earlier in training in the water T-maze.

The relative ease with which the rats solve the rotation place problem is somewhat surprising given the difficulty rats have in solving the translation place problem and the fact that there was no evidence of a place strategy on probe trials in Experiment 1. It has previously been argued that performance on these spatial tasks might be due to conditional discrimination learning where distinct start points (Skinner et al., 2003) or initial orientation/heading (Skinner, et al., in press; Wright, Williams, Evans, Skinner, \& Martin, 2009) guides responses at the choice point (i.e., if heading north, then make a right turn at the choice point). Rats in the Rotation group have distinct start points and initial orientation at the two maze positions (Figure 2) and learn as well as rats 
in the Response condition. Rats in the Translation group start from the same side of the room, making the initial heading the same and the start points less distinct, and are impaired relative to all other groups. The consistent pattern of data in appetitive and aversive situations suggests that similar strategies are used across tasks and motivational conditions.

\section{Future Directions}

The present research indicates that a place solution to a spatial problem is extremely difficult unless distinct start points are used. Rotation of the apparatus was key in making the start points distinct and thus allowing the animal to solve the problem. The use of transparent walls in the current apparatus did not seem to facilitate place learning, but this has not been systematically studied. A direct comparison between rats trained in a water T-maze with solid (opaque) walls and rats trained with transparent walls is needed. It remains to be seen whether probe trials conducted in an apparatus with transparent walls will reveal any evidence of place learning. Given the ease with which the Rotation rats acquired the task in Experiment 2, it is possible that probing animals in this group will reveal place learning.

In addition to recording trials to criterion in the acquisition experiment, conducting probe trials with those animals that did not solve the problem would also be informative. In the current experiment, no probe trials were given to animals that failed to reach criterion. Recording arm preference data in non-learners might prove useful since the initial probe data in Experiment 1 indicated that first choice is not always indicative of an animal's knowledge. During acquisition trials, animals are scored as making an error if they enter the arm without the platform. However, animals that chose a particular 
arm as their first choice on the probe trial often spent more time in the alternate arm over the $60 \mathrm{~s}$ probe trial.

Although cell-recoding studies have shown that place cells are often tied to the apparatus when the maze is translated but track distal cues when these are rotated, few of these studies have been conducted when the rats were actually solving spatial problems. In future experiments, cell recording could be done with rats trained on the rotation and translation place problems. Furthermore, comparisons between non-learners and those rats that are successful should prove interesting. 


\section{References}

Blodgett, H.C., \& McCutchen, K. (1948). Relative strength of place and response learning in the T-Maze. Journal of Comparative and Physiological Psychology, $41,17-24$.

Blodgett, H.C., McCutchen, K., \& Mathews, R. (1949). Spatial learning in the T-maze: The influence of direction, turn, and food location. Journal of Experimental Psychology, 39, $800-809$.

Chang, Q \& Gold, P.E. (2003). Switching memory systems during learning: Changes in patterns of brain acetylcholine release in the hippocampus and striatum of rats. The Journal of Neuroscience, 23, 3001-3005.

Hamilton, D.A., Akers, K.G., Johnson, T.E., Rice, J.P., Candelaria, F.T., \& Redhead, E.S. (2009). Evidence for a shift from place navigation to directional responding in one variant of the Morris water task. Journal of Experimental Psychology: Animal Behavior Processes, 35, 271 - 278.

Hamilton, D.A., Akers, K.G., Johnson, T.E., Rice, J.P., Candelaria, F.T., Sutherland, R.J., Weisend, M.P., \& Redhead, E.S. (2008). The relative influence of place and direction in the Morris water task. Journal of Experimental Psychology: Animal Behavior Processes, 34(1), 31 - 53.

Hamilton, D.A., Akers, K.G., Weisend, M.P., \& Sutherland, R.J. (2007). How do room and apparatus cues control navigation in the Morris water task? Evidence for distinct contributions to a movement vector. Journal of Experimental Psychology: Animal Behavior Processes, 33, 100 - 114. 
Horne, M.R., Martin, G.M., Harley, C.W. \& Skinner, D.M. (2007). Where am I? Distal cue use requires sensitivity to start location change in the rat. Journal of Experimental Psychology: Animal Behavior Processes, 33, 92-99.

Hull, C.L. (1934a). The concept of the habit-family hierarchy and maze learning. I. Psychological Review, 41, $33-54$.

Hull, C.L. (1934b). The concept of the habit-family hierarchy and maze learning. II. Psychological Review, 41, 34 - 52.

Hull, C.L. (1943). Principles of behavior. New York: Appleton-Century-Crofts.

Knierim, J.J. \& Rao, G. (2003). Distal landmarks and hippocampal place cells: Effects of relative translation versus rotation. Hippocampus, 13, 604-617.

Kubie, J.L., \& Ranck, J.B., Jr. (1983). Sensory-behavioral correlates in individual hippocampus neurons in three situations: Space and context. In W. Seifery (Ed). Neurobiology of the Hippocampus. Toronto, Ontario, Canada: Academic Press.

Morris, R.G.M. (1981). Spatial localization does not require the presence of local cues. Learning and Motivation, 12, $239-260$.

O.Keefe, J., \& Nadel, L. (1978). The hippocampus as a cognitive map. Oxford, England: Clarendon Press.

Packard, M.G. \& McGaugh, J.L. (1996). Inactivation of hippocampus or caudate nucleus with lidocaine differentially affects expression of place and response learning. Neurobiology of Learning and Memory, 65, 65 - 72.

Restle, F. (1957). Discrimination of cues in mazes: A resolution of the "place versus response" question. Psychological Review, 64, 217 - 228. 
Siegel, J.J., Rao, G., Lee, I., \& Knierim, J.J. (2005). Place cell representations in platform- versus room-defined coordinate frames in a spatial learning task. Society for Neuroscience Abstracts.

Skinner, D.M., Etchegary, C.M., Ekert-Maret, E.C., Baker, C.J., Harley, C.E., Evans, J.H., \& Martin, G.M. (2003). An analysis of response, direction, and place learning in an open field and T maze. Journal of Experimental Psychology: Animal Behavior Processes, 29, 3-13.

Skinner, D.M., Horne, M.R., Murphy, K.E.A., \& Martin, G.M. (in press). Rats' orientation is more important than start point location for successful place learning. Journal of Experimental Psychology: Animal Behavior Processes.

Stringer, K., Martin, G.M., \& Skinner, D.M. (2005). The effects of hippocampal lesions on response, direction, and place learning. Behavioral Neuroscience, 119, 946952.

Tolman, E.C. (1948). Cognitive maps in rats and men. Psychological Reviews, 55, 189 208.

Tolman, E.C., Ritchie, B.F., \& Kalish, D. (1946). Studies in spatial learning II: Place learning versus response learning. Journal of Experimental Psychology, 36, 221 229.

Walsh, S.J., Harley, C.W., Corbett, D., Skinner, D.M., \& Martin, G.M. (2008). CA1 ischemic injury does not affect the ability of Mongolian gerbils to solve response, direction, or place problems. Brain Research, 1187, 194-200.

Wright, S.L., Williams, D., Evans, J.H., Skinner, D., \& Martin, G.M. (2009). The contribution of spatial cues to memory: Direction, but not cue, changes support 
response reversal learning. Journal of Experimental Psychology: Animal Behavior Processes, 35, 177-185.

Woodward, R.S. (1938). Experimental Psychology. Henry Holt, New York.

Yoganarasimha, D., \& Knierim, J.J. (2005). Coupling between place cells and head direction cells during relative translations and rotations of distal landmarks. Experimental Brain Research, 160, $344-359$. 


\section{Figure Captions}

Figure 1: Schematic representations of Tolman, Ritchie \& Kalish's (1946) original maze positions.

Figure 2: Schematic representations of maze positions differentiating response, direction, and place strategies. Positions A - C show Blodgett, McCutchen, and Mathews (1949) original maze positions. Positions A - D show the positions used by Skinner et al. (2003), and in Experiment 1 of the present study. All eight positions show the training positions for half the rats in Experiment 2 of the present study. For example, in Experiment 2 half the rats in the Response group were trained to make a right turn (as indicated in the figure) while the other half were trained to make a left turn (not shown). Half the rats in the Direction group were trained to go east from positions B and D (as indicated in the figure) while the other half were trained to go west from positions $\mathrm{A}$ and $\mathrm{C}$ (not shown). The black bars indicate the barrier used to convert the plus maze to a Tmaze.

Figure 3: The top panel shows mean ( \pm SEM) trials correct over blocks of 8 trials for the four groups in experiment 1 . The bottom panel shows mean ( \pm SEM) latency (s) to locate the hidden platform over blocks of eight trials for the four groups in Experiment 1.

Figure 4: Maze positions during training and probe trials for half the animals in the four groups of experiment 1 . The other half of the rats in each group was trained to make the opposite response during training and the probe positions were changed accordingly. For example, half the rats in the Translation group were trained to make a right turn from one position (indicated in grey) and probed from a position (indicated in white) to the right of training. The other half of the rats were trained to make a left turn from the position 
indicated in white and probed from the position indicated in grey. The Translation test was an unambiguous test of place $(\mathrm{P})$ learning but confounded response $(\mathrm{R})$ and direction (D) strategies. The $90^{\circ}$ Rotation test assessed the strength of response and place strategies in the absence of a direction strategy. The $180^{\circ}$ Rotation test was an unambiguous test of response learning but confounded place and direction strategies. The maze was not moved during the probe trials for this group but the start arm was rotated by $180^{\circ}$. The Translation $+180^{\circ}$ Rotation test was an unambiguous test of direction learning but confounded place and response strategies. The numbers on the probe positions indicate the number of rats that chose a particular arm as their first choice; the letters indicate the strategy reflected in the arm choice. The black bars indicate the barrier used to convert the plus maze to a T-maze. The mean (+SEM) time (s) spent in the two arms are presented in the lower panels for each group.

Figure 5: Mean (+SEM) trials to criterion (18/20) for each of the 8 groups in Experiment 2. 
Response

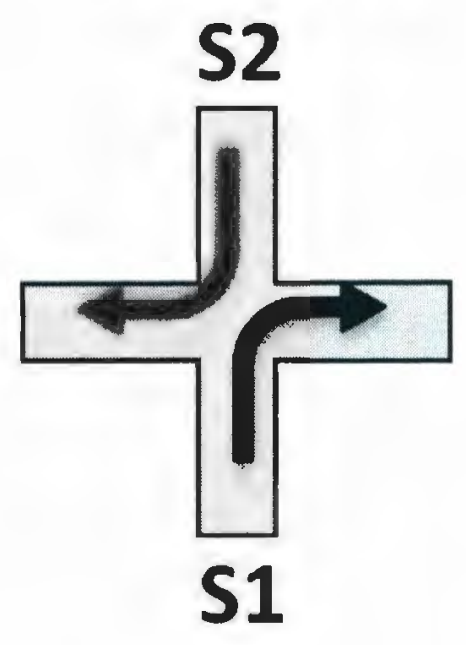

Place S2

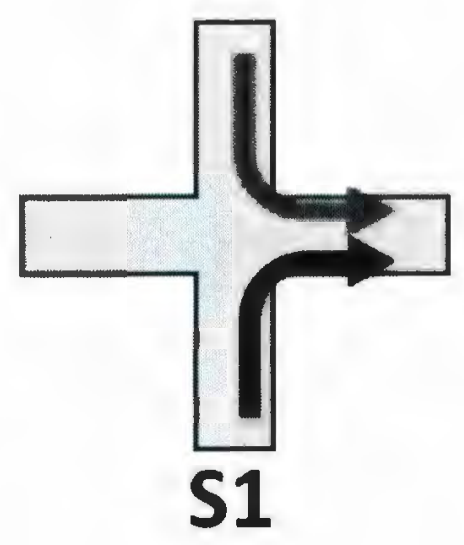


A.

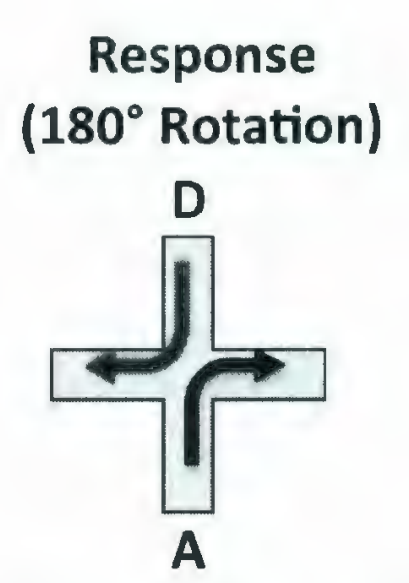

B.

Direction

(Translation $+180^{\circ}$ Rotation)

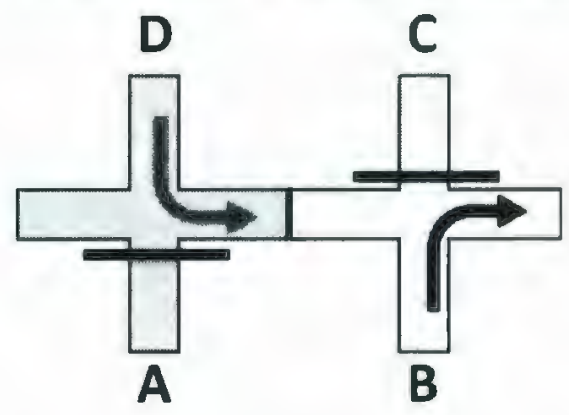

C. Translation (Place)

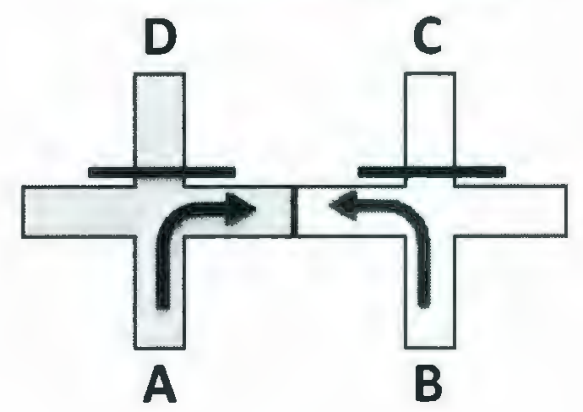

D. $90^{\circ}$ Rotation (Place)

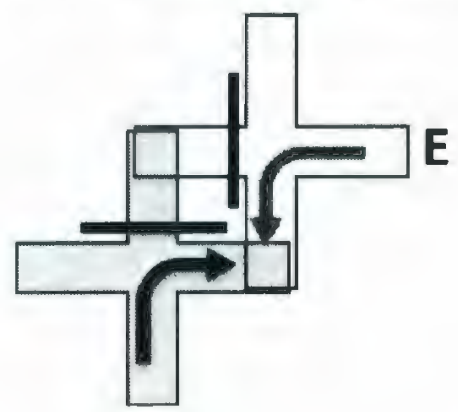

A

E. Place + Direction

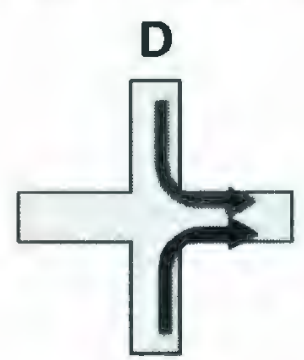

A
$\boldsymbol{F}$.

Place + Response

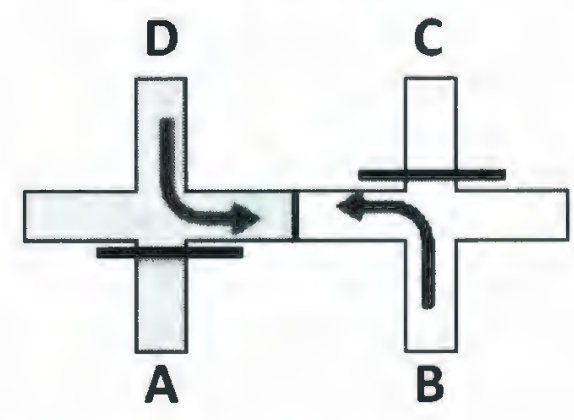

H. Response + Direction + Place

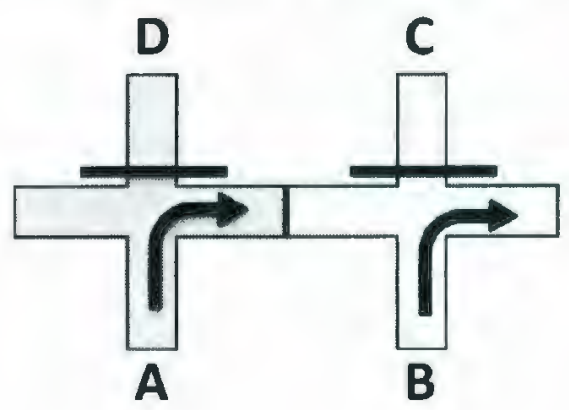

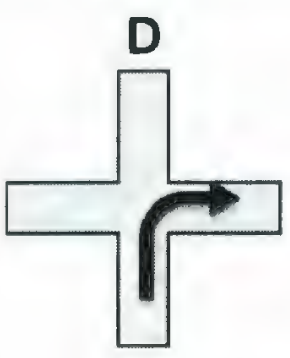

A 

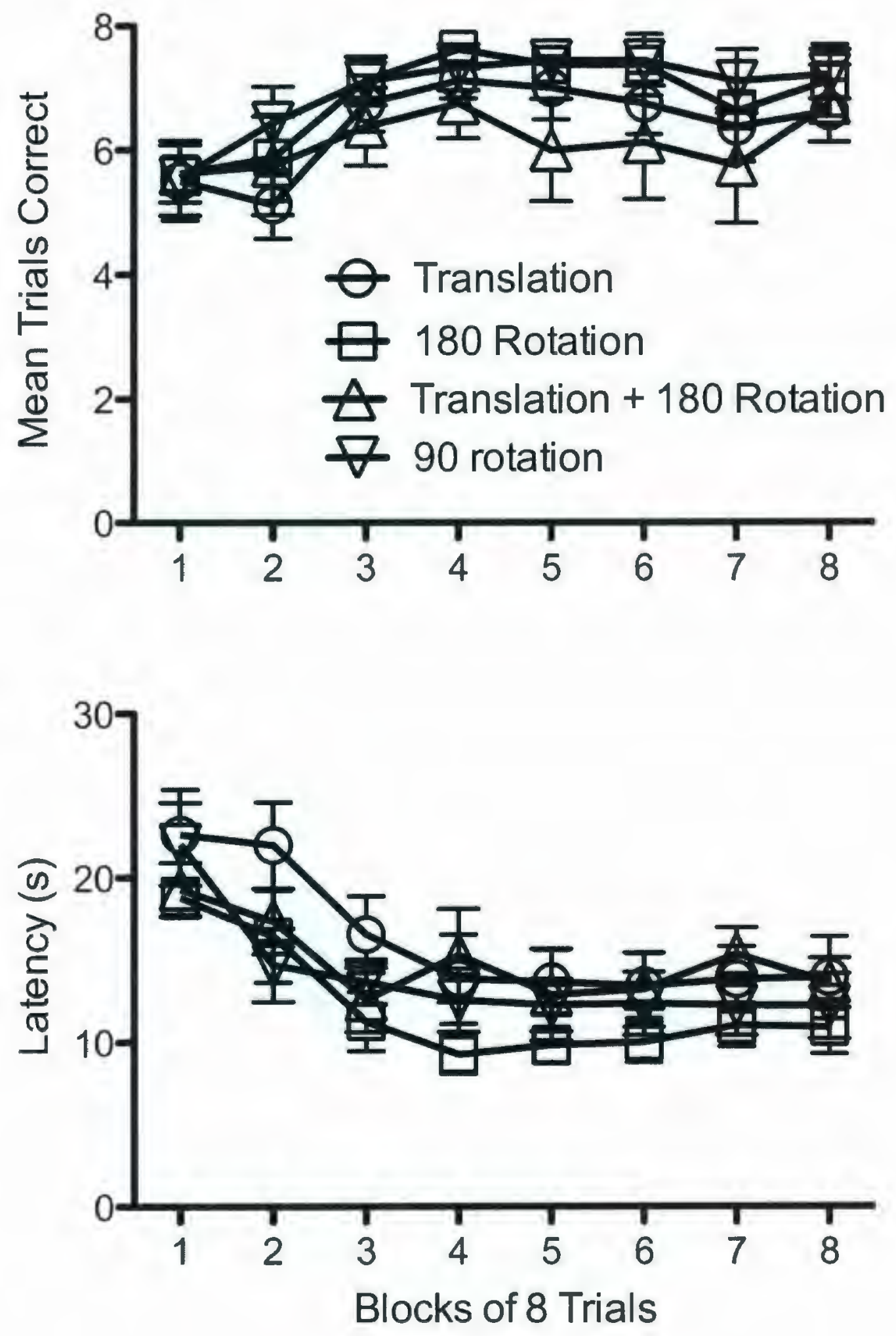
Translation
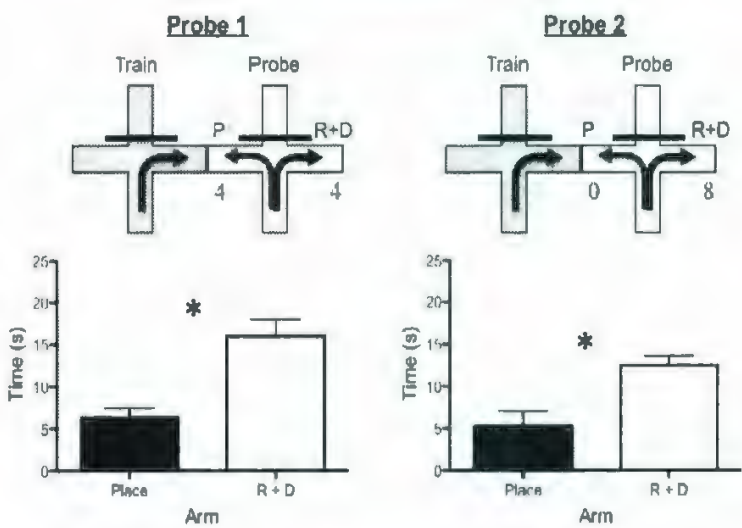

$180^{\circ}$ Rotation
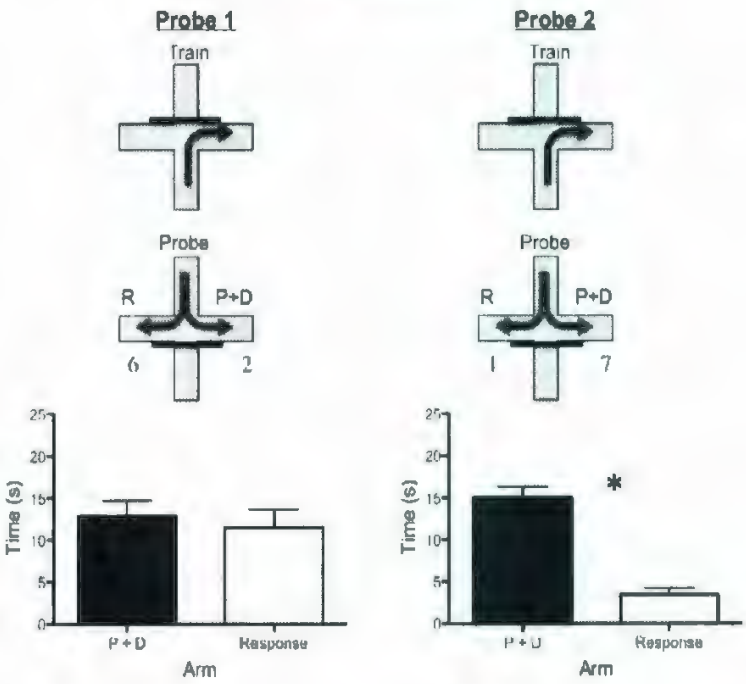

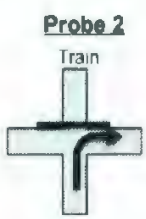

Probe?
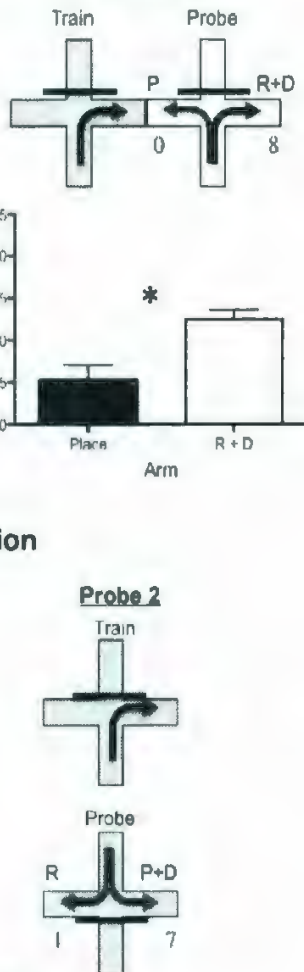

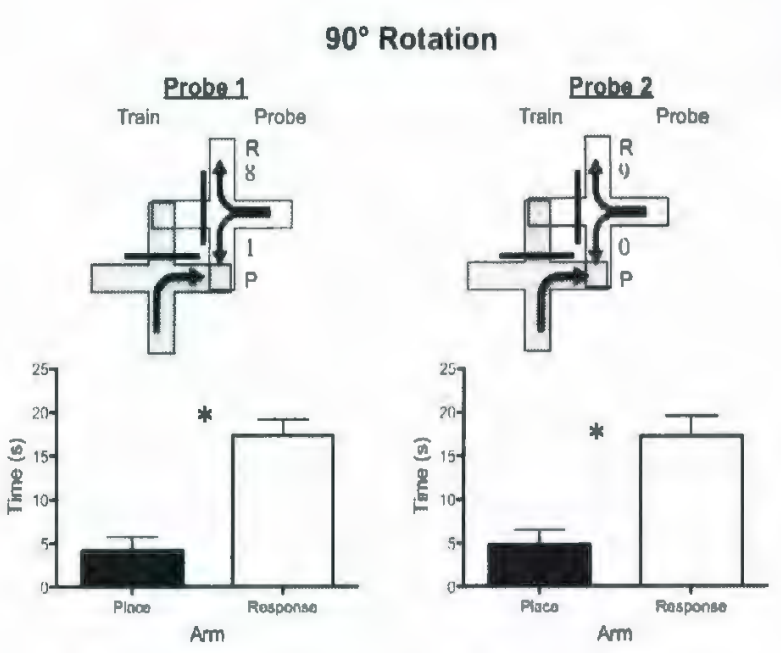

Translation $+180^{\circ}$ Rotation Probe 1

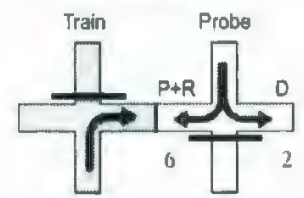
Probe?
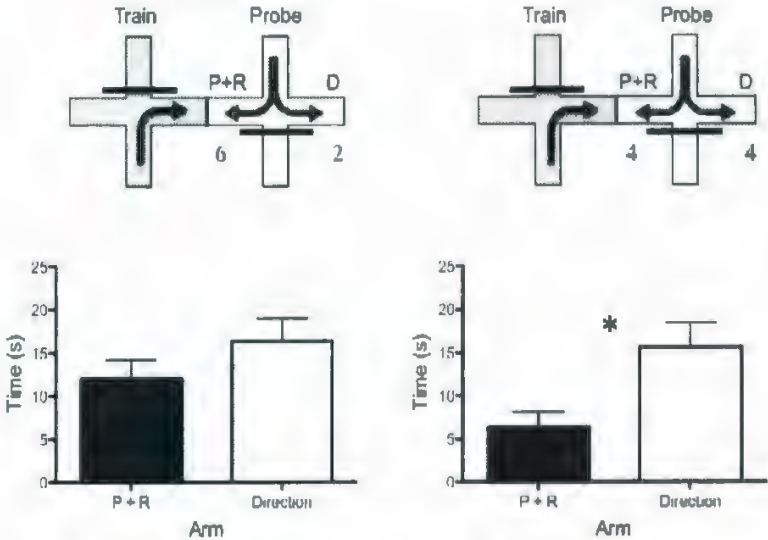
Place Learning 33

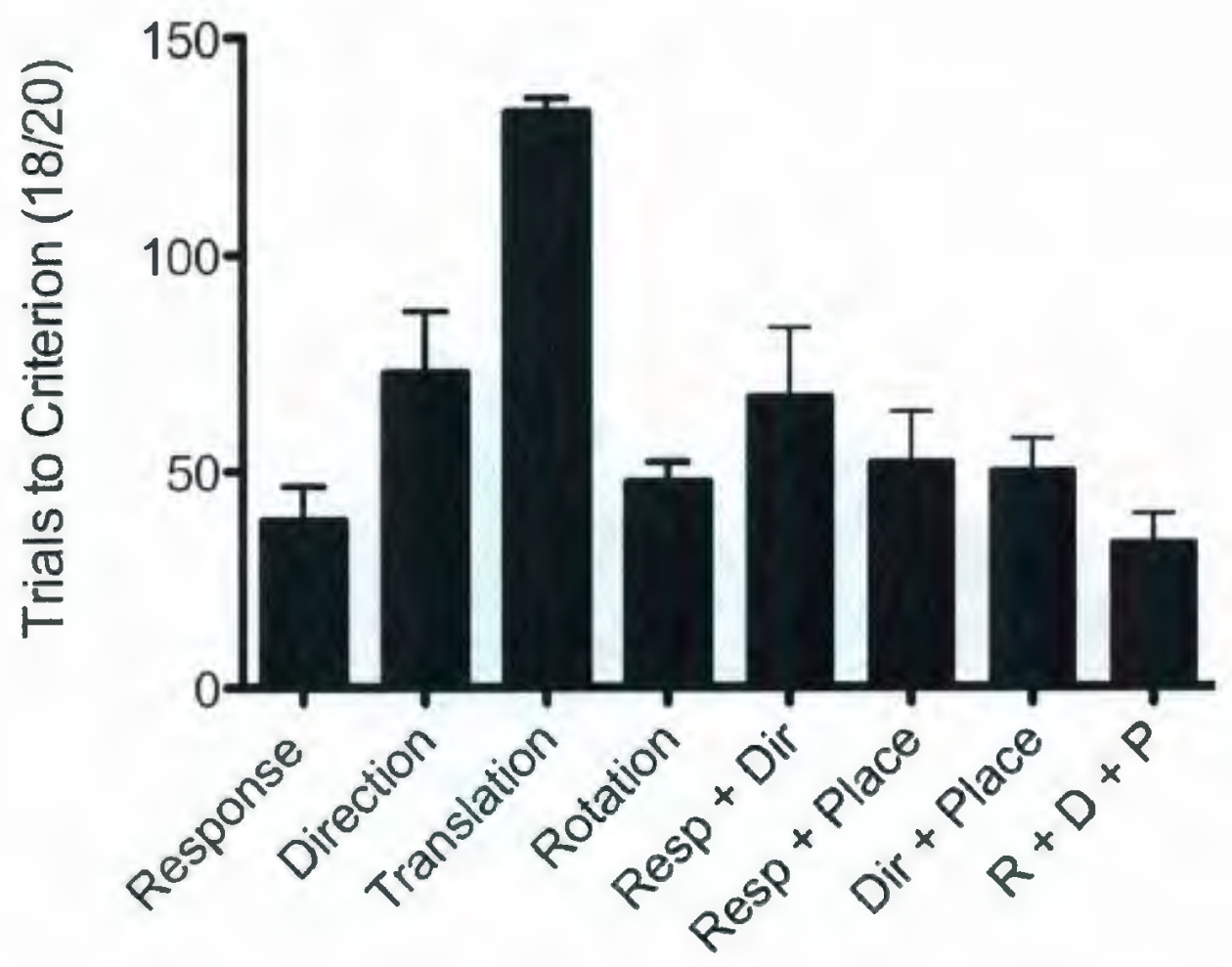

Group 

